\title{
Enthesitis in psoriatic arthritis
}

\author{
Mihaela Agache1, ${ }^{1,2}$, Catalina Elena Ionescul, ${ }^{1,2}$, Claudiu Popescu ${ }^{1,2}$, Luminita Enache ${ }^{1,2}$, \\ Corina Mogosan ${ }^{1,2}$, Catalin Codreanu ${ }^{1,2}$ \\ 1"Dr. Ion Stoia” Clinical Center for Rheumatic Diseases, Bucharest, Romania \\ 2"Carol Davila" University of Medicine and Pharmacy, Bucharest, Romania
}

Enthesitis is a distinctive feature of psoriatic arthritis. New imaging techniques help to better understand the pathophysiology of entheses, being one of the essential factors of the subclinical and prodromal stages of psoriatic arthritis. This paper aims to review the main clinical scores, imaging scores, confounding factors that might influence the interpretation of results and the impact of medication on enthesitis in psoriatic arthritis.

Keywords: psoriatic arthritis, enthesitis, ultrasound

\section{INTRODUCTION}

Psoriatic arthritis (PsA) is a chronic systemic inflammatory joint disease associated with psoriasis, included in the group of spondyloarthritis, characterized by inflammation of the joints, tendons, entheses or axial skeleton, with the potential for functional degradation (erosive and deforming arthritis). Baker first linked seronegative arthritis of the hands to psoriasis in 1963 [1]. The incidence of psoriatic arthritis is $0.3-1 \%$ of the populace with prevalence in distinct cohorts with psoriasis of 0.3-3.5/year [2]. Clinical manifestations include (in addition to skin and nails) dactylitis, spondylitis and peripheral manifestations: enthesitis, synovitis, bursitis, tenosynovitis and erosions.

Enthesitis is one of the characteristics of spondyloarthritis, it is a key manifestation of PsA, and current knowledge supports the concept that it could be among the primary events in the development of this disease [3]. Since 1966 Niepel has contributed to the initial clinical definitions of enthesopathy, describing anatomical involvement, clinical findings, radiographic findings, and histopathology [3]. The association of entesopathy with ankylosing spondylitis (AS) was made by Ball in 1970 [4]. The importance of enthesitis is recognized by its incorporation into the CASPAR criteria [5], but it is also one of the GRAPPA and OMERACT PSA core domains for dis- ease assessment along with peripheral arthritis, dactylitis and axial involvement [6]. Careful examination of each area by a rheumatologist is the first step to a correct diagnosis. Elevated acute-phase reactants and other extra-musculoskeletal features, such as the presence of uveitis, inflammatory bowel disease and nail psoriasis, help diagnose PsA.

\section{PATHOGENESIS}

If the pathogenic process of PsA was initially considered to target an antigen in the skin or joints, now studies show that the activation of the innate immune system as a result of external stimuli is responsible for clinical manifestations. Enthesitis is the proposed center of this model [7]. The entheses connect tendons and ligaments to bone through fibrocartilage, transmitting their mechanical force to the skeleton. The term "enthesis" comes from the ancient Greek (" $\varepsilon ้ v \theta \varepsilon \sigma \iota \varsigma$ ”) meaning "insertion".

The enthesal organ has an immune microenvironment that activates the innate immune system as a result of triggers: the most common is mechanical stress whose threshold can be determined genetically in order for the patient's response to the stimulus to be pathological $[8,9]$. The entheses contain fibers, fibroblasts, chondrocytes, cartilaginous matrix, mesenchymal cells with differentiation po- 
tential, chondrocytes and osteoblasts. Following mechanical stress, local mesenchymal cells produce prostaglandin E2 with a vasodilating and neutrophil recruitment effect in the bone marrow adjacent to the insertion of the entheses. Recruited macrophages and dendritic cells produce IL23. CD4 + $\mathrm{CD} 8+\mathrm{T}$ lymphocytes and locally-generated lymphoid cells have receptors for IL23 and produce IL22, IL17 and TNFa - cytokines that amplify the immune response [10,11]. Resident mesenchymal cells, under the influence of IL17, IL22 and IL23, proliferate and then differentiate into chondroblasts and osteoblasts, leading to periosteal bone apposition [11]. In 2007, McGonagle proposed the model of the synovio-enthesal complex [8]. The enthesal organ also includes the adjacent structures: fibrocartilage, fat pad, fascia.

The introduction of new treatments and the evolution of imaging methods allowed a better understanding of the pathology of entheses. For example, the presence of ultrasound power Doppler (PD) signals signifies an increase of local vascularization and it is a measure of local activity that revealed osteitis adjacent to the enthesis which can be observed on magnetic resonance imaging (MRI) [12].

Compared to synovitis, for which biopsies are feasible, the same cannot be achieved in the case of entheses, so imaging can be useful in identifying immunopathological mechanisms. Screening patients with cutaneous psoriasis using validated questionnaires could help early diagnosis, especially when associated with imaging. Delaying the diagnosis determines a worse prognosis [13].

\section{ENTHESES CLINICAL INDICES}

There is no consensus on clinical indices of enthesis. The most frequently used are LEI (Leeds Enthesitis Index), MASES (The Maastricht Ankylosing Spondylitis Enthesis Score) and SPARCC (Spondyloarthritis Research Consortium of Canada Index). LEI is the only score validated in PsA, while the others are used in spondyloarthritis. SPARCC is the closest to the data that can be obtained through current imaging techniques: ultrasound and MRI [14].

\section{TRANSITION PHASES FROM PSORIASIS TO PSA}

As in rheumatoid arthritis (RA), where there is a preclinical phase in the form of suspicious arthralgia associated with imaging signs (synovitis, tenosynovitis), so in PsA the existence of a preclinical phase is proposed. Ogdie et al. [2] emphasized in 2017 that psoriasis is an ideal disease for identifying risk factors for progression, because the patient pool is known. It is to be evaluated whether in pa- tients with psoriasis the musculoskeletal symptoms that present on imaging in the form of arthritis or enthesitis can predict the development of PsA. From a clinical point of view, Eder et al. [15] detected in 2017 nonspecific symptoms such as fatigue, stiffness and pain in patients with psoriasis who have progressed to PsA. The clinical examination hardly differentiates close anatomical structures. Ultrasound can achieve this, and it is important to differentiate the various anatomical structures because the treatment effectiveness may differ depending on the manifestations of the disease. In a study published in 2019, Zuliani et al. [16], using "active synovitis" or "active enthesitis" as a definition for subclinical PsA, included $40 \%$ of psoriasis patients without musculoskeletal manifestations in subclinical PsA. In 2020, Zabotti et al. [17] reported in patients with psoriasis and arthralgia that tenosynovitis can contribute significantly to symptoms, while synovitis and enthesitis can occur without symptoms. However, in this study enthesitis was a prognostic factor for PsA, which strengthens the role of enthesitis observed in experimental models. Scher et al. published in 2019 a theoretical model related to the progression from psoriasis to PsA [18]. The process begins with the interaction between genetic (and epigenetic) factors and exposure to environmental factors (such as biomechanical stress, infections, or obesity). The preclinical phase is characterized by aberrant activation of the immune system (activation of the IL-23 - IL-17 axis and TNF production). This phase is followed by a subclinical phase, in which imaging signs appear (ultrasound and MRI). Symptoms such as arthralgia and fatigue define the transition phase (also called the prodromal phase), which occurs prior to progression to clinically evident PsA. In the subclinical and prodromal phase, current ultrasound imaging techniques, MRI and computed tomography (CT) can identify inflammatory changes or changes in bone structure [19].

\section{IMAGING METHODS}

Conventional radiology can reveal in late stages of PsA changes in bone proliferation, it can detect erosions and it is useful for evaluating the spine and the sacroiliac joints.

Ultrasound has low reproducibility and cost. In 2018, OMERACT published the updated definition of enthesitis [20]: sonographic lesions that characterize enthesitis that include soft tissue structural lesions (entheseal thickening, hypoechogenicity and loss of tendon fibrillar pattern) and bone lesions (erosions, enthesophytes and calcifications) as well as the presence of neovascularization detected by Doppler signals. The intensity of the Doppler signal is quantified within a limit of $2 \mathrm{~mm}$ from the bone cortex [21]. 
The growing role of ultrasound in the diagnosis and monitoring of patients with PsA is also mentioned in the 2019 EULAR treatment recommendations, which give equal importance in PsA to both articular and peri-articular structures: enthesitis, tenosynovitis, dactylitis [22].

\section{ULTRASOUND SCORES}

Sonographic instruments for scoring enthesitis were developed for spondyloarthritis. None of them passed the OMERACT filter for PsA (the distribution of enthesitis in PsA may be different from spondyloarthritis [23]). Among the most used scores, the following can be mentioned [23]: Glasgow Ultrasound Enthesitis Scoring System (GUESS, which assesses 5 entheseal sites in the lower extremities); Madrid Sonographic Enthesitis Index (MASEI, which assesses 6 entheseal sites, incorporates lower and upper limb intents, and evaluates the presence of power Doppler); D 'Agostino score (which includes 10 enthesal areas, including two in the upper limbs); SEI (similar to GUESS, but it includes a distinction between chronic and acute lesions); Group for Assessment of Psoriasis and Psoriatic Arthritis (GRAPPA US: proposed entheseal sites by the GRAPPA Ultrasound Working Group) [24]; The Outcome Measures in Rheumatology OMERACT US: proposed entheseal sites by the OMERACT Ultrasound Enthesitis Working Group [20]; PsASon13 (a composite score which, besides joints, includes 2 enthesis [23]). As obesity and mechanical stress can interfere with lower limb entheses, it was necessary to integrate upper limb entheses into scores.

MRI reveals bone marrow edema and edema of soft tissues and enthesis structures, especially in the hand, where is not easy to differentiate them. An OMERACT score was published for the MRI evaluation of the small joints of the hands [25]. Recently, the HEMRIS score was created for the evaluation of heels [26]. There are studies of whole body MRI which can simultaneously evaluate peripheral damage and axial damage in patients with PsA and spondyloarthritis, by evaluating the distribution of inflammatory and structural lesions, producing a global entheseal score [27].

CT is useful in a later stage of the disease, when erosions and calcifications can be seen. High-resolution peripheral quantitative CT (HRqCT) is proposed to be used to detect new erosions and new bone formation in the hands. Similarly, dual energy CT (DECT)-iodine scans were superior to MRI in detecting lesions in the small joints of the hands. Research in this area was started by Fukuda in 2017 $[28,29]$ and continued by Shiraishi and Kayama in 2020 [30,31]. Derived from these, positron emission tomography CT (PET-CT) using fluorodeoxyglucose can detect changes in all symptomatic joints in 33\% of PsA patients [19].

Ultrasound remains the main method for diagnosis. Most studies in the literature use ultrasound as an imaging method in the diagnosis and monitoring of PsA. Ultrasound has high sensitivity and low cost, it is non-invasive, fast, reproducible, preferred by clinicians [32]. To guide the performance of ultrasound in patients with PsA in current clinical practice, TANG et al.[33] highlighted that in 120 patients with PsA and 320 patients with psoriasis, from a multitude of tendons, bursae and joints, the most commonly affected areas were: Achilles tendons, quadriceps tendons and knee joints, each with a weight of over $5 \%$. The most common ultrasound changes were the presence of Doppler signals in the enthesis and erosions at the insertion of the enthesis. In dactylitis, edema and thickening of subcutaneous cellular tissue can be observed, synovitis, pulley thickening, flexor and extensor enthesitis. Zabotti, considering all these, created a dactylitis score, DACTOS (DACTylitis glObal Sonographic score in PsA) [17]. Nail damage has been associated since 2006 with extraneous finger enthesitis [34]. In addition to the entheseal and synovial models, enthesitis causes inflammation of adjacent synovial structures. Thickening of the A1 anchoring system in the flexor tendon (pulley - a small and relatively vascular structure) with local positive Doppler signals is also associated with PsA, the pulley being a functional enthesis [35-37]. ABRAR et al. also demonstrates that MRI evaluation of flexor tendon pulley may be a distinctive marker for PsA compared to RA or a control group [35, 38]. Power Doppler is the most valuable of the parameters analyzed at the level of enthesis [39, 40], both MASEI score and the OMERACT power Doppler scores detected $75 \%$ power Doppler signals in patients with active disease $[39,40]$.

\section{PREVALENCE OF SUBCLINICAL MUSCULOSKELETAL SIGNS}

There are several studies that show changes in the enthesis of patients with psoriasis without joint symptoms that suggest that they are the first site of inflammation. Instead, there are few studies that detect lesions that cause progression from psoriasis to PsA. Zuliani et al. found in 2019 in 40 patients with psoriasis a prevalence of $27 \%$ of synovitis and a prevalence of $20 \%$ of active enthesitis, which were included in subclinical PsA [41]. Savage et al. in 2020 in a group of patients with psoriasis without musculoskeletal signs, found that $49.3 \%$ of the subjects presented elements that met the OMERACT definition for enthesopathy [42]. In another study, Vyas et al. evaluated 50 patients with psoriasis and 
50 control patients, reporting that $62 \%$ of patients with psoriasis had subclinical enthesopathy compared with only $10 \%$ of controls [43]. The mean MASES score between psoriasis and control cases was statistically significant with the setting of a cutoff of 11 that can differentiate between a patient with clinically significant enthesopathy and those with non-inflammatory enthesopathy. Yen et al. in 2021, using ultrasound imaging, managed to diagnose $47 \%$ of psoriasis patients with preclinical stages of PsA [44]. There are also differences in the thickness of the entheses between patients with PsA and those with psoriasis, the greatest thickness was found in enthesis with erosions. Increasing thickness is considered the first accurate sign of inflammation [45]. It is necessary to evaluate longitudinally how many of these psoriasis patients develop definite PsA, in order to assess which of these lesions is more specific for risk stratification. Savage et al. proposed that a Leeds model of evaluation should be used by the dermatologist-rheumatologist team in order to capture the early stages of PsA [42]. Rida et al. also proposed a way to evaluate patients at risk: psoriasis patients require screening with questionnaires, evaluation by imaging methods, and when clinical manifestations occur [13], clinical examination, imaging and evaluation of inflammation parameters are recommended.

In most studies, there was low agreement between clinical scores and imaging scores, but ultrasound was found to be more specific in detecting preclinical lesions [46,47]. Clinical evaluation of painful sites may detect subtle damage to tendons, bursae, erosions, enthesophytes, calcifications, but it may be interfered by concomitant causes for pain (e. g. fibromyalgia). The exclusion of fibromyalgia was studied intensely in the last decade [48], since clinical examinations can overestimate the extent of joint damage/inflammation in patients who also have fibromyalgia. Ultrasound was studied in patients with PsA and PsA-fibromyalgia compared to those with fibromyalgia alone, and it was found useful in differentiating the intensity of PsA-related pain from fibromyalgia $[49,50]$. There are other biomechanical confounding factors that can cause symptoms such as obesity and menstrual stress [51]. Also, in the control groups, the thickening of the entheses was most frequently noted, but no there were no power Doppler signals detected [52]. Matteo et al. tried to estimate how prevalent it is in healthy subjects in a recently published paper. The authors evaluated 82 healthy subjects, applying the definition of OMERACT, and found an increased prevalence of $34 \%$ of subjects with entheasal damage and $8.4 \%$ of entheses (820 entheses were evaluated), correlating them with age and body mass in$\operatorname{dex}[52,53]$.

\section{PREDICTORS OF PSA}

Zaboti et al. reported in 2019 that enthesitis was associated with progression to PsA [16]. Ultrasound-visualized enthesophytes predict their radiological progression, representing a severity marker [54]. The severity of sonographic enthesitis scores, including inflammatory scores and damage scores, was associated with higher radiographic damage scores in the peripheral and axial joints [55]. This aspect is also confirmed by the recent study published by Marcia-Villa et al. in 2021 [56]: MASEI was associated with axial and articular radiological damage. It was found that patients with psoriasis with subclinical inflammation on MRI and arthralgia had a high risk (55.5\%) for the development of PsA, while patients without arthralgia had a low risk (15.3\%) for the development of PsA [57].

The US GRAPPA is working to develop a scoring system (DUET) to distinguish PsA from other non-inflammatory diseases using musculoskeletal ultrasound and to evaluate its correlation with clinical measures of disease activity (enthesitis count, joint number) and patient-reported outcomes [58]. Although $50 \%$ of patients with psoriasis have subclinical changes, only some of them progress to PsA. This progression is influenced by the type of psoriasis (nail, scalp, inverted), obesity and genetics (grade 1 relatives with arthritis).

\section{PSA TREATMENT CAN IMPROVE THE ULTRASOUND SIGNS OF ENTHESITIS}

Treatment with adalimumab of patients with psoriasis and enthesitis showed a decrease in quadriceps tendon thickening at weeks 24 and 48 [59]. In the 2021 study by Collada et al. [39], the power Doppler signal decreases significantly at 3-6 months of biological therapy on a group of 25 patients with spondyloarthritis and PsA. A study of ustekinumab in patients with psoriasis is also reported: intensity scores decreased by $42 \%$ at week 24 and by $47.5 \%$ at week 42 [60]. The fact that psoriasis treatment can prevent the development of PsA is shown in another study of 20 patients with psoriasis in which $83 \%$ had subclinical enthesitis or synovitis and after 24 weeks of treatment with secukinumab, the PaAMRIS score and synovitis scores were significantly improved. On the other hand, erosions and enthesophytes did not progress and the total bone mass at the level of the distal radius increased after 24 weeks.

The recent Delphi Consensus emphasizes that the terms "preclinical" or "prodromal" can only be used retrospectively and tried to find agreement on the definition of these patients for inclusion criteria in prospective trials to quantify the risk of progres- 
sion. The strategy is to evaluate patients with psoriasis with moderate damage, including scalp or nails, or with predisposing genetic traits. Sarabia et al. suggested to combine ultrasound with a screening questionnaire to improve the triage of these patients [61].

\section{REFERENCES}

1. Baker H, Golding DN, Thompson M. Psoriasis and arthritis. $\mathrm{Br}$ Med J. 1963;2(5371):1527.

2. Ogdie A. The preclinical phase of PsA: a challenge for the epidemiologist. Ann Rheum Dis. 2017;1481-1483.

3. Niepel GA. Enthesopathy. Acta rheumatologica et balneologica Pistiniana, 1966.

4. Ball J. Enthesopathy of rheumatoid and ankylosing spondylitis. Ann Rheum Dis. 1971;30(3):213-23.

5. Taylor W, et al. Classification criteria for psoriatic arthritis: development of new criteria from a large international study. Arthritis Rheum. 2006;54(8):2665-73.

6. Gladman DD, et al. Consensus on a core set of domains for psoriatic arthritis. J Rheumatol. 2007;34(5):1167-70.

7. Paulissen SM, et al. Synovial fibroblasts directly induce Th17 pathogenicity via the cyclooxygenase/prostaglandin E2 pathway, independent of IL-23. J Immunol. 2013;191(3):1364-72.

8. McGonagle D, et al. The concept of a „synovio-entheseal complex" and its implications for understanding joint inflammation and damage in psoriatic arthritis and beyond. Arthritis Rheum. 2007;56(8):2482-91.

9. Benjamin M, Evans EJ, Copp L. The histology of tendon attachments to bone in man. J Anat. 1986;149:89-100.

10. Sherlock JP, et al. IL-23 induces spondyloarthropathy by acting on ROR- $y t+C D 3+C D 4-C D 8$ - entheseal resident T cells. Nat Med. 2012;18(7):1069-76.

11. Gravallese EM, Schett G. Effects of the IL-23-IL-17 pathway on bone in spondyloarthritis. Nat Rev Rheumatol. 2018;14(11):631640.

12. Schett G, et al. Enthesitis: from pathophysiology to treatment. Nat Rev Rheumatol. 2017;13(12):731-741.

13. Rida MA, Chandran V. Challenges in the clinical diagnosis of psoriatic arthritis. Clin Immunol. 2020;214:108390.

14. Bakewell C, et al. Imaging Techniques: Options for the Diagnosis and Monitoring of Treatment of Enthesitis in Psoriatic Arthritis. J Rheumatol. 2020;47(7):973-982.

15. Eder, L, et al. The Development of Psoriatic Arthritis in Patients With Psoriasis Is Preceded by a Period of Nonspecific Musculoskeletal Symptoms: A Prospective Cohort Study. Arthritis Rheumatol. 2017;69(3):622-629.

16. Zabotti A, et al. Transition phase towards psoriatic arthritis: clinical and ultrasonographic characterisation of psoriatic arthralgia. RMD Open. 2019;5(2):e001067.

17. Zabotti A, et al. From Psoriasis to Psoriatic Arthritis: Insights from Imaging on the Transition to Psoriatic Arthritis and Implications for Arthritis Prevention. Curr Rheumatol Rep. 2020;22(6):24.

18. Scher JU, et al. Preventing psoriatic arthritis: focusing on patients with psoriasis at increased risk of transition. Nat Rev Rheumatol. 2019;15(3):153-166.

19. Köhm M, et al. Innovative Imaging Technique for Visualization of Vascularization and Established Methods for Detection of Musculoskeletal Inflammation in Psoriasis Patients. Front Med (Lausanne). 2020;7:468.

20. 20. Balint PV, et al. Reliability of a consensus-based ultrasound definition and scoring for enthesitis in spondyloarthritis and psoriatic arthritis: an OMERACT US initiative. Ann Rheum Dis. 2018;77(12):1730-1735.

21. Terslev L, et al. The OMERACT Stepwise Approach to Select and Develop Imaging Outcome Measurement Instruments:

\section{CONCLUSION}

All these data suggest that early treatment of the disease in the preclinical phases of PsA could possibly lead to a decline in subclinical inflammation preventing the occurrence of PsA in high-risk individuals.

The Musculoskeletal Ultrasound Example. J Rheumatol. 2019; 46(10):1394-1400.

22. Gossec L, et al. EULAR recommendations for the management of psoriatic arthritis with pharmacological therapies: 2019 update. Ann Rheum Dis. 2020;79(6):700-712.

23. Elalouf $O$, et al. Psoriatic Arthritis Sonographic Enthesitis Instruments: A Systematic Review of the Literature. J Rheumatol. 2019;46(1):43-56.

24. Tom S, et al. Development of a Preliminary Ultrasonographic Enthesitis Score in Psoriatic Arthritis - GRAPPA Ultrasound Working Group. J Rheumatol. 2019;46(4):384-390.

25. Mathew AJ, et al. The OMERACT MRI in Enthesitis Initiative: Definitions of Key Pathologies, Suggested MRI Sequences, and a Novel Heel Enthesitis Scoring System. I Rheumatol. 2019;46(9):1232-1238.

26. Mathew AJ, et al. Atlas of the OMERACT Heel Enthesitis MRI Scoring System (HEMRIS). RMD Open. 2020;6(1).

27. Poggenborg RP, et al. Head-to-toe whole-body MRI in psoriatic arthritis, axial spondyloarthritis and healthy subjects: first steps towards global inflammation and damage scores of peripheral and axial joints. Rheumatology (Oxford). 2015;54(6):1039-49.

28. Fukuda T, et al. Initial Experience of Using Dual-Energy CT with an lodine Overlay Image for Hand Psoriatic Arthritis: Comparison Study with Contrast-enhanced MR Imaging. Radiology. 2017;284(1):134-142.

29. Fukuda $T$, et al. Dual energy CT iodine map for delineating inflammation of inflammatory arthritis. Eur Radiol. 2017;27(12): 5034-5040.

30. Shiraishi $M$, et al. Differentiating Rheumatoid and Psoriatic Arthritis of the Hand: Multimodality Imaging Characteristics. Radiographics. 2020;40(5):1339-1354.

31. Kayama R, et al. Quantitative analysis of therapeutic response in psoriatic arthritis of digital joints with Dual-energy CT iodine maps. Sci Rep. 2020;10(1):1225.

32. Grassi W, Salaffi F, Filippucci E. Ultrasound in rheumatology. Best Pract Res Clin Rheumatol. 2005;19(3):467-85.

33. Tang $\mathrm{Y}$, et al. Ultrasound assessment in psoriatic arthritis (PsA) and psoriasis vulgaris (non-PsA): which sites are most commonly involved and what features are more important in PsA? Quant Imaging Med Surg. 2020;10(1):86-95.

34. Ash ZR, et al. Psoriasis patients with nail disease have a greater magnitude of underlying systemic subclinical enthesopathy than those with normal nails. Ann Rheum Dis. 2012;71(4):553-6.

35. Tinazzi I, et al. Power Doppler enhancement of accessory pulleys confirming disease localization in psoriatic dactylitis. Rheumatology (Oxford). 2020;59(8):2030-2034.

36. Tinazzi I, et al. ,Deep Koebner' phenomenon of the flexor tendon-associated accessory pulleys as a novel factor in tenosynovitis and dactylitis in psoriatic arthritis. Ann Rheum Dis. 2018;77(6):922-925.

37. Tinazzi l, et al. The early psoriatic arthritis screening questionnaire: a simple and fast method for the identification of arthritis in patients with psoriasis. Rheumatology (Oxford). 2012;51(11):2058-63.

38. Abrar DB, et al. High-resolution MRI of flexor tendon pulleys using a 16-channel hand coil: disease detection and differentiation of psoriatic and rheumatoid arthritis. Arthritis Res Ther. 2020;22(1):40. 
39. Molina Collada J, et al. Ultrasound Doppler enthesitis shows sensitivity to change after biological therapy in spondyloarthritis and psoriatic arthritis patients. Scand J Rheumatol. 2021:1-9.

40. Molina Collada J, et al. Doppler enthesitis: a potential useful outcome in the assessment of axial spondyloarthritis and psoriatic arthritis. Clin Rheumatol. 2021;40(5):2013-2020.

41. Zuliani $F$, et al. Ultrasonographic detection of subclinical enthesitis and synovitis: a possible stratification of psoriatic patients without clinical musculoskeletal involvement. Clin Exp Rheumatol. 2019;37(4):593-599.

42. Savage L, et al. Defining Pre-Clinical Psoriatic Arthritis in an Integrated Dermato-Rheumatology Environment. J Clin Med. 2020;9(10).

43. Vyas K, et al. Sonographic Evaluation of Subclinical Enthesopathy in Patients of Chronic Plaque Psoriasis. Indian Dermatol Online J. 2020;11(4):580-585.

44. Yen TH, et al. Ultrasound-aided diagnosis of preclinical phases of psoriatic arthritis in biologic-naïve psoriasis patients with or without arthralgia. Clin Exp Rheumatol. 2021.

45. Graceffa D, et al. Ultrasound assessment of enthesis thickness in psoriasis and psoriatic arthritis: A cross-sectional study. Indian J Dermatol Venereol Leprol. 2019;85(2):175-181.

46. Yamada $Y$, et al. Ultrasound assessment, unlike clinical assessment, reflects enthesitis in patients with psoriatic arthritis. Clin Exp Rheumatol. 2021;39(1):139-145.

47. Perrotta FM, et al. An ultrasonographic study of enthesis in early psoriatic arthritis patients naive to traditional and biologic DMARDs treatment. Rheumatol Int. 2016;36(11):1579-1583.

48. Fiorenza A, et al. Assessment of enthesis in patients with psoriatic arthritis and fibromyalgia using clinical examination and ultrasound. Clin Exp Rheumatol. 2020;38 Suppl 123(1):31-39.

49. Marchesoni A, et al. The problem in differentiation between psoriatic-related polyenthesitis and fibromyalgia. Rheumatology (Oxford). 2018;57(1):32-40.

50. Macchioni P, et al. Ultrasonographic and Clinical Assessment of Peripheral Enthesitis in Patients with Psoriatic Arthritis, Psoriasis, and Fibromyalgia Syndrome: The ULISSE Study. J Rheumatol. 2019;46(8):904-911.

Conflict of interest: none declared

Financial support: none declared
51. Kaeley GS. Enthesitis in psoriatic arthritis (Part 2): imaging. Rheumatology (Oxford). 2020;59(Supplement_1):i15-i20.

52. Di Matteo A, et al. How normal is the enthesis by ultrasound in healthy subjects? Clin Exp Rheumatol. 2020;38(3):472-478.

53. Mathew AJ et al. Psoriatic arthritis: lessons from imaging studies and implications for therapy. Expert Rev Clin Immunol. 2017;13(2):133-142.

54. Lackner A, et al. Ultrasound verified enthesophytes are associated with radiographic progression at entheses in psoriatic arthritis. Rheumatology (Oxford). 2020;59(10):2893-2897.

55. Polachek A, et al. The association between sonographic enthesitis and radiographic damage in psoriatic arthritis. Arthritis Res Ther. 2017;19(1):189.

56. Macía-Villa C, Cruz Valenciano A, De Miguel E, Enthesis lesions are associated with $\mathrm{X}$-ray progression in psoriatic arthritis. Int $\mathrm{J}$ Rheum Dis. 2021;24(6):828-833.

57. Faustini $F$, et al. Subclinical joint inflammation in patients with psoriasis without concomitant psoriatic arthritis: a cross-sectional and longitudinal analysis. Ann Rheum Dis. 2016;75(12):20682074.

58. Eder L, Kaeley GS, Aydin SZ. Development and Validation of a Sonographic Enthesitis Instrument in Psoriatic Arthritis: The GRAPPA Diagnostic Ultrasound Enthesitis Tool (DUET) Project. J Rheumatol Suppl. 2020 Jun;96:50-52.

59. Narcisi, A, et al. Effects of TNF- $\alpha$ inhibition on pre-clinical enthesitis: observational study on 49 psoriatic patients. J Dermatolog Treat. 2021:1-4.

60. Savage, L, et al. Regression of Peripheral Subclinical Enthesopathy in Therapy-Naive Patients Treated With Ustekinumab for Moderate-to-Severe Chronic Plaque Psoriasis: A Fifty-TwoWeek, Prospective, Open-Label Feasibility Study. Arthritis Rheumatol. 2019;71(4):626-631.

61. Sarabia, S, et al. Comparative Efficacy of Different Triage Methods for Psoriatic Arthritis - Prospective Study in a Rapid Access Clinic. Arthritis Care Res (Hoboken). 2021. 